\title{
Sliding Mode Approach to Control Quadrotor Using Dynamic Inversion
}

\author{
Abhijit Das, Frank L. Lewis and Kamesh Subbarao \\ Automation and Robotics Research Institute \\ The University of Texas at Arlington \\ USA
}

\section{Introduction}

Nowadays unmanned rotorcraft are designed to operate with greater agility, rapid maneuvering, and are capable of work in degraded environments such as wind gusts etc. The control of this rotorcraft is a subject of research especially in applications such as rescue, surveillance, inspection, mapping etc. For these applications, the ability of the rotorcraft to maneuver sharply and hover precisely is important (Koo and Sastry 1998). Rotorcraft control as in these applications often requires holding a particular trimmed state; generally hover, as well as making changes of velocity and acceleration in a desired way (Gavrilets, Mettler, and Feron 2003). Similar to aircraft control, rotorcraft control too involves controlling the pitch, yaw, and roll motion. But the main difference is that, due to the unique body structure of rotorcraft (as well as the rotor dynamics and other rotating elements) the pitch, yaw and roll dynamics are strongly coupled. Therefore, it is difficult to design a decoupled control law of sound structure that stabilizes the faster and slower dynamics simultaneously. On the contrary, for a fixed wing aircraft it is relatively easy to design decoupled standard control laws with intuitively comprehensible structure and guaranteed performance (Stevens and F. L. Lewis 2003). There are many different approaches available for rotorcraft control such as (Altug, Ostrowski, and Mahony 2002; Bijnens et al. 2005; T. Madani and Benallegue 2006; Mistler, Benallegue, and M'Sirdi 2001; Mokhtari, Benallegue, and Orlov 2006) etc. Popular methods include input-output linearization and back-stepping. The 6-DOF airframe dynamics of a typical quadrotor involves the typical translational and rotational dynamical equations as in (Gavrilets, Mettler, and Feron 2003; Castillo, Lozano, and Dzul 2005; Castillo, Dzul, and Lozano 2004). The dynamics of a quadrotor is essentially a simplified form of helicopter dynamics that exhibits the basic problems including underactuation, strong coupling, multi-input/multi-output, and unknown nonlinearities. The quadrotor is classified as a rotorcraft where lift is derived from the four rotors. Most often they are classified as helicopters as its movements are characterized by the resultant force and moments of the four rotors. Therefore the control algorithms designed for a quadrotor could be applied to a helicopter with relatively straightforward modifications. Most of the papers (B. Bijnens et al. 2005; T. Madani and Benallegue 2006; Mokhtari, Benallegue, and Orlov 2006) etc. deal with either input-output linearization for decoupling pitch yaw roll or back-stepping to deal with the under-actuation problem. The problem of coupling in the 
yaw-pitch-roll of a helicopter, as well as the problem of coupled dynamics-kinematic underactuated system, can be solved by back-stepping (Kanellakopoulos, Kokotovic, and Morse 1991; Khalil 2002; Slotine and Li 1991). Dynamic inversion (Stevens and F. L. Lewis 2003; Slotine and Li 1991; A. Das et al. 2004) is effective in the control of both linear and nonlinear systems and involves an inner inversion loop (similar to feedback linearization) which results in tracking if the residual or internal dynamics is stable. Typical usage requires the selection of the output control variables so that the internal dynamics is guaranteed to be stable. This implies that the tracking control cannot always be guaranteed for the original outputs of interest.

The application of dynamic inversion on UAV's and other flying vehicles such as missiles, fighter aircrafts etc. are proposed in several research works such as (Kim and Calise 1997; Prasad and Calise 1999; Calise et al. 1994) etc. It is also shown that the inclusion of dynamic neural network for estimating the dynamic inversion errors can improve the controller stability and tracking performance. Some other papers such as (Hovakimyan et al. 2001; Rysdyk and Calise 2005; Wise et al. 1999; Campos, F. L. Lewis, and Selmic 2000) etc. discuss the application of dynamic inversion on nonlinear systems to tackle the model and parametric uncertainties using neural nets. It is also shown that a reconfigurable control law can be designed for fighter aircrafts using neural net and dynamic inversion. Sometimes the inverse transformations required in dynamic inversion or feedback linearization are computed by neural network to reduce the inversion error by online learning.

In this chapter we apply dynamic inversion to tackle the coupling in quadrotor dynamics which is in fact an underactuated system. Dynamic inversion is applied to the inner loop, which yields internal dynamics that are not necessarily stable. Instead of redesigning the output control variables to guarantee stability of the internal dynamics, we use a sliding mode approach to stabilize the internal dynamics. This yields a two-loop structured tracking controller with a dynamic inversion inner loop and an internal dynamics stabilization outer loop. But it is interesting to notice that unlike normal two loop structure, we designed an inner loop which controls and stabilizes altitude and attitude of the quadrotor and an outer loop which controls and stabilizes the position $(x, y)$ of the quadrotor. This yields a new structure of the autopilot in contrast to the conventional loop linear or nonlinear autopilot. Section 2 of this chapter discusses the basic quadrotor dynamics which is used for control law formulation. Section 3 shows dynamic inversion of a nonlinear state-space model of a quadrotor. Sections 4 discuss the robust control method using sliding mode approach to stabilize the internal dynamics. In the final section, simulation results are shown to validate the control law discussed in this chapter.

\section{Quadrotor dynamics}

Fig. 1 shows a basic model of an unmanned quadrotor. The quadrotor has some basic advantage over the conventional helicopter. Given that the front and the rear motors rotate counter-clockwise while the other two rotate clockwise, gyroscopic effects and aerodynamic torques tend to cancel in trimmed flight. This four-rotor rotorcraft does not have a swashplate (P. Castillo, R. Lozano, and A. Dzul 2005). In fact it does not need any blade pitch control. The collective input (or throttle input) is the sum of the thrusts of each motor (see Fig. 1). Pitch movement is obtained by increasing (reducing) the speed of the rear motor while reducing (increasing) the speed of the front motor. The roll movement is obtained similarly using the lateral motors. The yaw movement is obtained by increasing (decreasing) 
the speed of the front and rear motors while decreasing (increasing) the speed of the lateral motors (Bouabdallah, Noth, and Siegwart 2004).

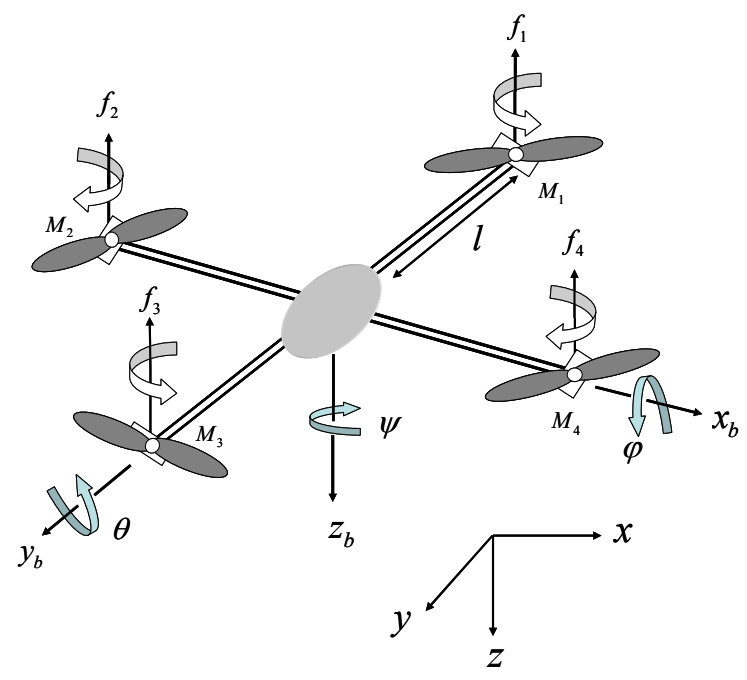

Fig. 1. A typical model of a quadrotor helicopter

In this section we will describe the basic state-space model of the quadrotor. The dynamics of the four rotors are relatively much faster than the main system and thus neglected in our case. The generalized coordinates of the rotorcraft are $q=(x, y, z, \psi, \theta, \varphi)$, where $(x, y, z)$ represents the relative position of the center of mass of the quadrotor with respect to an inertial frame $\mathfrak{I}$, and $(\psi, \theta, \varphi)$ are the three Euler angles representing the orientation of the rotorcraft, namely yaw-pitch-roll of the vehicle.

Let us assume that the transitional and rotational coordinates are in the form $\xi=(x, y, z)^{T} \in R^{3}$ and $\eta=(\psi, \theta, \varphi) \in R^{3}$. Now the total transitional kinetic energy of the rotorcraft will be $T_{\text {trans }}=\frac{m}{2} \dot{\xi}^{\mathrm{T}} \dot{\xi}$ where $m$ is the mass of the quadrotor. The rotational kinetic energy is described as $T_{r o t}=\frac{1}{2} \dot{\eta}^{T} J \dot{\eta}$, where matrix $J=J(\eta)$ is the auxiliary matrix expressed in terms of the generalized coordinates $\eta$. The potential energy in the system can be characterized by the gravitational potential, described as $U=m g z$. Defining the Lagrangian $L=T_{\text {trans }}+T_{\text {rot }}-U$, where $T_{\text {trans }}=(m / 2) \dot{\xi}^{T} \dot{\xi}$ is the translational kinetic energy, $T_{\text {rot }}=(1 / 2) \omega^{T} I \omega$ is the rotational kinetic energy with $\omega$ as angular speed, $U=m g z$ is the potential energy, $z$ is the quadrotor altitude, $I$ is the body inertia matrix, and $g$ is the acceleration due to gravity.

Then the full quadrotor dynamics is obtained as a function of the external generalized forces $F=\left(F_{\xi}, \tau\right)$ as

$$
\frac{d}{d t} \frac{\partial L}{\partial \dot{q}}-\frac{\partial L}{\partial q}=F
$$


The principal control inputs are defined as follows. Define

$$
F_{R}=\left(\begin{array}{l}
0 \\
0 \\
u
\end{array}\right)
$$

where $u$ is the main thrust and defined by

$$
u=f_{1}+f_{2}+f_{3}+f_{4}
$$

and $f_{i}^{\prime}$ s are described as $f_{i}=k_{i} \omega_{i}^{2}$, where $k_{i}$ are positive constants and $\omega_{i}$ are the angular speed of the motor $i$. Then $F_{\xi}$ can be written as

$$
F_{\xi}=\bar{R} F_{R}
$$

where $\bar{R}$ is the transformation matrix representing the orientation of the rotorcraft as

$$
\bar{R}=\left(\begin{array}{ccc}
c_{\theta} c_{\psi} & s_{\psi} s_{\theta} & -s_{\theta} \\
c_{\psi} s_{\theta} s_{\varphi}-s_{\psi} c_{\varphi} & s_{\psi} s_{\theta} s_{\varphi}+c_{\psi} c_{\varphi} & c_{\theta} s_{\varphi} \\
c_{\psi} s_{\theta} c_{\varphi}+s_{\psi} s_{\varphi} & s_{\psi} s_{\theta} c_{\varphi}-c_{\psi} s_{\varphi} & c_{\theta} c_{\varphi}
\end{array}\right)
$$

The generalized torque for the $\eta$ variables are

where

$$
\tau=\left(\begin{array}{c}
\tau_{\psi} \\
\tau_{\theta} \\
\tau_{\varphi}
\end{array}\right)
$$

$$
\begin{aligned}
\tau_{\psi}=\sum_{i=1}^{4} \tau_{M_{i}} & =c\left(f_{1}-f_{2}+f_{3}-f_{4}\right) \\
\tau_{\theta} & =\left(f_{2}-f_{4}\right) l \\
\tau_{\varphi} & =\left(f_{3}-f_{1}\right) l
\end{aligned}
$$

Thus the control distribution from the four actuator motors of the quadrotor is given by

$$
\left(\begin{array}{c}
u \\
\tau_{\varphi} \\
\tau_{\theta} \\
\tau_{\psi}
\end{array}\right)=\underbrace{\left(\begin{array}{cccc}
1 & 1 & 1 & 1 \\
-l & 0 & l & 0 \\
0 & l & 0 & -l \\
c & -c & c & -c
\end{array}\right)}_{C} \underbrace{\left(\begin{array}{l}
f_{1} \\
f_{2} \\
f_{3} \\
f_{4}
\end{array}\right)}_{\underline{f}}
$$

where $l$ is the distance from the motors to the center of gravity, $\tau_{M_{i}}$ is the torque produced by motor $M_{i}$, and $c$ is a constant known as force-to-moment scaling factor. So, if a required thrust and torque vector are given, one may solve for the rotor force using (10). 
The final dynamic model of the quadrotor is described by (11)-(14),

$$
\begin{gathered}
m \ddot{\xi}+\left(\begin{array}{c}
0 \\
0 \\
m g
\end{array}\right)=F_{R} \\
J(\eta) \ddot{\eta}+\frac{d}{d t}\{J(\eta)\} \dot{\eta}-\frac{1}{2} \frac{\partial}{\partial \eta}\left(\dot{\eta}^{T} J(\eta) \dot{\eta}\right)=\tau \\
J(\eta) \ddot{\eta}+\frac{d}{d t}\{J(\eta)\} \dot{\eta}-\bar{C}(\eta, \dot{\eta})=\tau \\
J(\eta) \ddot{\eta}+C(\eta, \dot{\eta})=\tau
\end{gathered}
$$

where, $F_{R}=u\left(\begin{array}{c}-\sin \theta \\ \cos \theta \sin \varphi \\ \cos \theta \cos \varphi\end{array}\right)$, auxiliary Matrix $\{J(\eta)\}=J=T_{\eta}^{T} I T_{\eta}$ with

$$
T_{\eta}=\left(\begin{array}{ccc}
-\sin \theta & 0 & 1 \\
\cos \theta \sin \psi & \cos \psi & 0 \\
\cos \theta \cos \psi & -\sin \psi & 0
\end{array}\right)
$$

Now finally the dynamic model of the quadrotor in terms of position $(x, y, z)$ and rotation $(\varphi, \theta, \psi)$ is written as,

$$
\begin{aligned}
& \left(\begin{array}{c}
\ddot{x} \\
\ddot{y} \\
\ddot{z}
\end{array}\right)=\left(\begin{array}{c}
0 \\
0 \\
-g
\end{array}\right)+\frac{1}{m}\left(\begin{array}{c}
-\sin \theta \\
\cos \theta \sin \varphi \\
\cos \theta \cos \varphi
\end{array}\right) u \\
& \left(\begin{array}{c}
\ddot{\varphi} \\
\ddot{\theta} \\
\ddot{\psi}
\end{array}\right)=f(\varphi, \theta, \psi)+g(\varphi, \theta, \psi) \tau
\end{aligned}
$$

where,

$$
f(\varphi, \theta, \psi)=\left(\begin{array}{c}
\dot{\theta} \dot{\psi}\left(\frac{I_{y}-I_{z}}{I_{x}}\right)-\frac{J_{p}}{I_{x}} \dot{\theta} \Omega \\
\dot{\varphi} \dot{\psi}\left(\frac{I_{z}-I_{x}}{I_{y}}\right)+\frac{J_{p}}{I_{y}} \dot{\varphi} \Omega \\
\dot{\varphi} \dot{\theta}\left(\frac{I_{x}-I_{y}}{I_{z}}\right)
\end{array}\right), g(\varphi, \theta, \psi)=\left(\begin{array}{ccc}
\frac{l}{I_{x}} & 0 & 0 \\
0 & \frac{l}{I_{y}} & 0 \\
0 & 0 & \frac{l}{I_{z}}
\end{array}\right), u \in R^{1} \text { and } \tau=\left[\begin{array}{c}
\tau_{\varphi} \\
\tau_{\theta} \\
\tau_{\psi}
\end{array}\right] \in R^{3} \text { are the }
$$

control inputs, $I_{x, y, z}$ are body inertia, $J_{p}$ is propeller/rotor inertia and $\Omega=\omega_{2}+\omega_{4}-\omega_{1}-\omega_{3}$. Thus, the system is the form of an under-actuated system with six outputs and four inputs. 
Comment 2.1: In this chapter we considered a generalized state space model of quadrotor derived from Lagrangian dynamics. Design autopilot with actual Lagrangian model of quadrotor is discussed in (Abhijit Das, Frank Lewis, and Kamesh Subbarao 2009).

\section{Partial feedback linearization for Quadrotor model}

Dynamic inversion (Stevens and F. L. Lewis 2003) is an approach where a feedback linearization loop is applied to the tracking outputs of interest. The residual dynamics, not directly controlled, is known as the internal dynamics. If the internal dynamics are stable, dynamic inversion is successful. Typical usage requires the selection of the output control variables so that the internal dynamics is guaranteed to be stable. This means that tracking cannot always be guaranteed for the original outputs of interest.

In this chapter we apply dynamic inversion to the system given by (15) and (16) to achieve station-keeping tracking control for the position outputs $(x, y, z, \psi)$. Initially we select the convenient output vector $y_{d i}=(z, \varphi, \theta, \psi)$ which makes the dynamic inverse easy to find. Dynamic inversion now yields effectively an inner control loop that feedback linearizes the system from the control $u_{d i}=\left(u, \tau_{\varphi}, \tau_{\theta}, \tau_{\psi}\right)$ to the output $y_{d i}=(z, \varphi, \theta, \psi)$. Note that the output contains attitude parameters as well as altitude of the quadrotor.

Note however that $y_{d i}$ is not the desired system output. Moreover, dynamic inversion generates a specific internal dynamics, as detailed below, which may not always be stable. Therefore, a second outer loop is designed to generate the required values for $y_{d i}=(z, \varphi, \theta, \psi)$ in terms of the values of the desired tracking output $(x, y, z, \psi)$. An overall Lyapunov proof guarantees stability and performance. The following background is required. Consider a nonlinear system of the form

$$
\dot{q}=f\left(q, u_{q}\right)
$$

where $u_{q} \in R^{m}$ is the control input and $q \in R^{n}$ is state vector. The technique of designing the control input $u$ using dynamic inversion involves two steps. First, one finds a state transformation $z=z(q)$ and an input transformation $u_{q}=u_{q}(q, v)$ so that the nonlinear system dynamics is transformed into an equivalent linear time invariant dynamics of the form

$$
\dot{z}=a z+b v
$$

where $a \in R^{n \times n}, b \in R^{n \times m}$ are constant matrices with $v$ is known as new input to the linear system. Secondly one can design $v$ easily from the linear control theory approach such as pole placement etc. To get the desired linear equations (18), one has to differentiate outputs until input vector $u_{d i}$ appears. The procedure is known as dynamic inversion.

\subsection{Dynamic inversion for inner loop}

The system, (15) $\rightarrow(16)$ is an underactuated system if we consider the states $(x, y, z, \varphi, \theta, \psi)$ as outputs and $u_{d i}=\left[\begin{array}{llll}u & \tau_{\varphi} & \tau_{\theta} & \tau_{\psi}\end{array}\right]^{T}$ as inputs. To overcome these difficulties we consider four outputs $y_{d i}=(z, \varphi, \theta, \psi)$ which are used for feedback linearization. Differentiating the output vector twice with respect to the time we get from (15) and (16) that, 


$$
\ddot{y}_{d i}=M_{d i}+E_{d i} u_{d i}
$$

where,

$$
M_{d i}=\left[\begin{array}{c}
\dot{\theta} \dot{\psi}\left(\frac{I_{y}-I_{z}}{I_{x}}\right)-\frac{J_{p}}{I_{x}} \dot{\theta} \Omega \\
\dot{\varphi} \dot{\psi}\left(\frac{I_{z}-I_{x}}{I_{y}}\right)+\frac{J_{p}}{I_{y}} \dot{\varphi} \Omega \\
\dot{\varphi} \dot{\theta}\left(\frac{I_{x}-I_{y}}{I_{z}}\right)
\end{array}\right] \in \mathfrak{R}^{4}, E_{d i}=\left[\begin{array}{cccc}
-(1 / m) \cos \theta \cos \varphi & 0 & 0 & 0 \\
0 & \frac{l}{I_{x}} & 0 & 0 \\
0 & 0 & \frac{l}{I_{y}} & 0 \\
0 & 0 & 0 & \frac{l}{I_{z}}
\end{array}\right] \in \mathfrak{R}^{4 \times 4}
$$

The number $(r=8)$ of differentiation required for an invertible $E_{d i}$ is known as the relative degree of the system and generally $r<n=12$; if $r=n$ then full state feedback linearization is achieved if $E_{d i}$ is invertible. Note that for multi-input multi-output system, if number of outputs is not equal to the number of inputs (under-actuated system), then $E_{d i}$ becomes non-square and is difficult to obtain a feasible linearizing input $u_{d i}$.

It is seen that for non-singularity of $E_{d i}, 0 \leq \theta, \varphi<90^{\circ}$. The relative degree of the system is calculated as 8 whereas the order of the system is 12 . So, the remaining dynamics $(=4)$ which does not come out in the process of feedback linearization is known as internal dynamics. To guarantee the stability of the whole system, it is mandatory to guarantee the stability of the internal dynamics. In the next section we will discuss how to control the internal dynamics using a PID with a feed-forward acceleration outer loop. Now using (19) we can write the desired input to the system

$$
u_{d i}=E_{d i}^{-1}\left(-M_{d i}+v_{d i}\right)
$$

which yields

$$
\ddot{y}_{d i}=v_{d i}
$$

where, $v_{d i}=\left(\begin{array}{llll}v_{z} & v_{\varphi} & v_{\theta} & v_{\psi}\end{array}\right)^{T}$. This system is decoupled and linear. The auxiliary input $v_{d i}$ is designed as described below.

\subsection{Design of linear controller}

Assuming the desired output to the system is $y_{d}=\left(\begin{array}{llll}z_{d} & \varphi_{d} & \theta_{d} & \psi_{d}\end{array}\right)^{T}$, the linear controller $v_{d i}$ is designed in the following way

$$
v_{d i}=\left[\begin{array}{c}
v_{z} \\
v_{\varphi} \\
v_{\theta} \\
v_{\psi}
\end{array}\right]=\left[\begin{array}{c}
\ddot{z}_{d}-K_{1_{z}}\left(\dot{z}-\dot{z}_{d}\right)-K_{2_{z}}\left(z-z_{d}\right) \\
\ddot{\varphi}_{d}-K_{1_{\varphi}}\left(\dot{\varphi}-\dot{\varphi}_{d}\right)-K_{2_{\varphi}}\left(\varphi-\varphi_{d}\right) \\
\ddot{\theta}_{d}-K_{1_{\theta}}\left(\dot{\theta}-\dot{\theta}_{d}\right)-K_{2_{\theta}}\left(\theta-\theta_{d}\right) \\
\ddot{\psi}_{d}-K_{1_{\psi}}\left(\dot{\psi}-\dot{\psi}_{d}\right)-K_{2_{\psi}}\left(\psi-\psi_{d}\right)
\end{array}\right]
$$


where, $K_{1_{\varphi}}, K_{2_{\rho}}, \ldots$ etc. are positive constants so that the poles of the error dynamics arising from (23) and (24) are in the left half of the $s$-plane. For hovering control, $z_{d}$ and $\psi_{d}$ are chosen depending upon the designer choice.

\subsection{Defining sliding variable error}

Let us define the state error $e_{1}=\left(\begin{array}{llll}z_{d}-z & \varphi_{d}-\varphi & \theta_{d}-\theta & \psi_{d}-\psi\end{array}\right)^{T}$ and a sliding mode error as

$$
r_{1}=\dot{e}_{1}+\Lambda_{1} e_{1}
$$

where, $\Lambda_{1}$ is a diagonal positive definite design parameter matrix. Common usage is to select $\Lambda_{1}$ diagonal with positive entries. Then, (23) is a stable system so that $e_{1}$ is bounded as long as the controller guarantees that the filtered error $r_{1}$ is bounded. In fact it is easy to show (F. Lewis, Jagannathan, and Yesildirek 1999) that one has

$$
\left\|e_{1}\right\| \leq \frac{\left\|r_{1}\right\|}{\sigma_{\min }\left(\Lambda_{1}\right)},\left\|\dot{e}_{1}\right\| \leq\left\|r_{1}\right\|
$$

Note that $\dot{e}_{1}+\Lambda_{1} e_{1}=0$ defines a stable sliding mode surface. The function of the controller to be designed is to force the system onto this surface by making $r_{1}$ small. The parameter $\Lambda_{1}$ is selected for a desired sliding mode response

$$
e_{1}(t)=e_{1}^{-\Lambda_{1} t} e_{1}(0)
$$

We now focus on designing a controller to keep $\left\|r_{1}\right\|$ small. From (23),

$$
\dot{r}_{1}=\ddot{e}_{1}+\Lambda_{1} \dot{e}_{1}
$$

Adding an integrator to the linear controller given in (22), and now we can rewrite (22) as

$$
v_{d i}=\ddot{y}_{d i_{d}}+K_{1} \dot{e}_{1}+K_{2} e_{1}+K_{3} \int_{0}^{t} r_{1} d t
$$

where, $y_{d i_{d}}=\left[\ddot{z}_{d}, \ddot{\varphi}_{d}, \ddot{\theta}_{d}, \ddot{\psi}_{d}\right]^{T}$ and $K_{i}=\operatorname{diag}\left(K_{i_{z}}, K_{i_{\varphi}}, K_{i_{\theta}}, K_{i_{\psi}}\right)>0, i=1,2,3,4$.

Now using equation (20) and (27) we can rewrite the equation (19) in the form of error dynamics as

$$
\ddot{e}_{1}+K_{1} \dot{e}_{1}+K_{2} e_{1}+K_{3} \int_{0}^{t} r_{1} d t=0
$$

Thus equation (26) becomes

$$
\dot{r}_{1}=-K_{1} \dot{e}_{1}-K_{2} e_{1}-K_{3} \int_{0}^{t} r_{1} d t+\Lambda_{1} \dot{e}_{1}
$$

If we choose $K_{1}=\left(\Lambda_{1}+R\right), K_{2}=\Lambda_{1} R$, then equation (29) will look like

$$
\dot{r}_{1}=-R r_{1}-K_{3} \int_{0}^{t} r_{1} d t
$$


Note that $R>0$ is also a diagonal matrix.

\section{Sliding mode control for internal dynamics}

The internal dynamics (Slotine and Li 1991) for the feedback linearizes system given by

$$
\begin{gathered}
\ddot{x}=-\frac{u}{m} \sin \theta \\
\ddot{y}=\frac{u}{m} \cos \theta \sin \varphi
\end{gathered}
$$

For the stability of the whole system as well as for the tracking purposes, $x, y$ should be bounded and controlled in a desired way. Note that the altitude $z$ of the rotorcraft a any given time $t$ is controlled by (20),(22).

To stabilize the zero dynamics, we select some desired $\theta_{d}$ and $\varphi_{d}$ such that $(x, y)$ is bounded. Then that $\left(\theta_{d}, \varphi_{d}\right)$ can be fed into (22) as a reference. Using Taylor series expansion about some nominal values $\theta_{d}^{*}, \varphi_{d}^{*}$ and considering up to first order terms

$$
\begin{aligned}
& \sin \theta_{d}=\sin \theta_{d}^{*}+\cos \theta_{d}^{*}\left(\theta_{d}-\theta_{d}^{*}\right) \\
& \cos \theta_{d}=\cos \theta_{d}^{*}-\sin \theta_{d}^{*}\left(\theta_{d}-\theta_{d}^{*}\right) \\
& \sin \varphi_{d}=\sin \varphi_{d}^{*}+\cos \varphi_{d}^{*}\left(\varphi_{d}-\varphi_{d}^{*}\right)
\end{aligned}
$$

Using (33) on (31) we get

$$
\begin{gathered}
\ddot{x}=-\frac{u}{m}\left\{\sin \theta_{d}^{*}+\cos \theta_{d}^{*}\left(\theta_{d}-\theta_{d}^{*}\right)\right\} \\
\ddot{y}=\frac{u}{m}\left\{\cos \theta_{d}^{*}-\sin \theta_{d}^{*}\left(\theta_{d}-\theta_{d}^{*}\right)\right\}\left\{\sin \varphi_{d}^{*}+\cos \varphi_{d}^{*}\left(\varphi_{d}-\varphi_{d}^{*}\right)\right\}
\end{gathered}
$$

For hovering of a quadrotor, assuming the nominal values $\theta_{d}^{*} \approx 0, \varphi_{d}^{*} \approx 0,(31)$ and (32) becomes

$$
\begin{gathered}
\ddot{x}=-\frac{u}{m} \theta_{d} \\
\ddot{y}=\frac{u}{m} \varphi_{d}
\end{gathered}
$$

Define the state error

$$
e_{2}=\left(\begin{array}{ll}
x_{d}-x & y_{d}-y
\end{array}\right)^{T}
$$

and the sliding mode error for the internal dynamics as

$$
r_{2}=\dot{e}_{2}+\Lambda_{2} e_{2}
$$


where, $\Lambda_{2}$ is a diagonal positive definite design parameter matrix with similar characteristic of $\Lambda_{1}$. Also

$$
\left\|e_{2}\right\| \leq \frac{\left\|r_{2}\right\|}{\sigma_{\min }\left(\Lambda_{2}\right)},\left\|\dot{e}_{2}\right\| \leq\left\|r_{2}\right\|
$$

Therefore according to (40), designing a controller to keep $\left\|r_{2}\right\|$ small will guarantee that $\left\|e_{2}\right\|$ and $\left\|\dot{e}_{2}\right\|$ are small. Differentiating $r_{2}$ we get

$$
\dot{r}_{2}=\ddot{e}_{2}+\Lambda_{2} \dot{e}_{2}
$$

Let the choice of the control law is as follows

$$
\begin{aligned}
& \theta_{d}=\frac{m}{u}\left[-\ddot{x}_{d}-c_{11}\left(\dot{x}_{d}-\dot{x}\right)-c_{12}\left(x_{d}-x\right)-c_{13} \int_{0}^{t} r_{2} d t-\beta_{x} \operatorname{sgn}\left(r_{2}\right)\right], \beta_{x}>0 \\
& \varphi_{d}=\frac{m}{u}\left[\ddot{y}_{d}+c_{21}\left(\dot{y}_{d}-\dot{y}\right)+c_{22}\left(y_{d}-y\right)+c_{23} \int_{0}^{t} r_{2} d t+\beta_{y} \operatorname{sgn}\left(r_{2}\right)\right], \beta_{y}>0
\end{aligned}
$$

where, $\left[\begin{array}{cc}c_{11} & 0 \\ 0 & c_{21}\end{array}\right]=C_{1}>0,\left[\begin{array}{cc}c_{12} & 0 \\ 0 & c_{22}\end{array}\right]=C_{2}>0,\left[\begin{array}{cc}c_{13} & 0 \\ 0 & c_{23}\end{array}\right]=C_{3}>0$ and $\left[\begin{array}{cc}\beta_{x} & 0 \\ 0 & \beta_{y}\end{array}\right]=\beta>0$.

Combining the equations (36) to (43)

$$
\ddot{e}_{2}+C_{1} \dot{e}_{2}+C_{2} e_{2}+C_{3} \int_{0}^{t} r_{2} d t+\beta \operatorname{sgn}\left(r_{2}\right)=0
$$

Therefore

$$
\dot{r}_{2}=-C_{1} \dot{e}_{2}-C_{2} e_{2}-C_{3} \int_{0}^{t} r_{2} d t-\beta \operatorname{sgn}\left(r_{2}\right)+\Lambda_{2} \dot{e}_{2}
$$

Let

$$
\begin{gathered}
C_{1}=\Lambda_{2}+S_{0} \\
C_{2}=\Lambda_{2} S_{0}
\end{gathered}
$$

Therefore

$$
\begin{gathered}
\dot{r}_{2}=-\left(\Lambda_{2}+S_{0}\right) \dot{e}_{2}-\Lambda_{2} S_{0} e_{2}-C_{3} \int_{0}^{t} r_{2} d t-\beta \operatorname{sgn}\left(r_{2}\right)+\Lambda_{2} \dot{e}_{2} \\
\dot{r}_{2}=-S_{0} r_{2}-C_{3} \int_{0}^{t} r_{2} d t-\beta \operatorname{sgn}\left(r_{2}\right)
\end{gathered}
$$




\section{Controller structure and stability analysis}

The overall control system has two loops and is depicted in Fig. 2. The following theorem details the performance of the controller.

Definition 5.1: The equilibrium point $x_{e}$ is said to be uniformly ultimately bounded (UUB) if there exist a compact set $S \subset R^{n}$ so that for all $x_{0} \in S$ there exist a bound $B$ and a time $T\left(B, x_{0}\right)$ such that $\left\|x(t)-x_{e}(t)\right\| \leq B \forall t \geq t_{0}+T$.

Theorem 5.1: Given the system as described in (15) and (16) with a control law shown in Fig. 2. and given by (20), (27), (42), (43). Then, the tracking errors $r_{1}$ and $r_{2}$ and thereby $e_{1}$ and $e_{2}$ are UUB if (53) and (54) are satisfied and can be made arbitrarily small with a suitable choice of gain parameters. According to the definition given by (23) of $r_{1}$ and (39) of $r_{2}$, this guarantees that $e_{1}$ and $e_{2}$ are $U U B$ since

$$
\begin{array}{ll}
\left\|e_{1}\right\| \leq \frac{\left\|r_{1}\right\|}{\sigma_{\text {min }}\left(\Lambda_{1}\right)} \leq \frac{b_{r_{1}}}{\sigma_{\text {min }}\left(\Lambda_{1}\right)} & b_{r_{1}}>0 \\
\left\|e_{2}\right\| \leq \frac{\left\|r_{2}\right\|}{\sigma_{\text {min }}\left(\Lambda_{2}\right)} \leq \frac{b_{r_{2}}}{\sigma_{\text {min }}\left(\Lambda_{2}\right)} & b_{r_{2}}>0
\end{array}
$$

where $\sigma_{\min }\left(\Lambda_{i}\right)$ is the minimum singular value of $\Lambda_{i}, i=1,2$.

Proof: Consider the Lyapunov function

$$
L=\frac{1}{2} r_{1}^{T} P_{1} r_{1}+\frac{1}{2} r_{2}^{T} Q_{1} r_{2}+\frac{1}{2}\left[\int_{0}^{t} r_{1}^{T} d t\right] P_{2}\left[\int_{0}^{t} r_{1} d t\right]+\frac{1}{2}\left[\int_{0}^{t} r_{2}^{T} d t\right] Q_{2}\left[\int_{0}^{t} r_{2} d t\right]
$$

with symmetric matrices $P_{1}, P_{2}, Q_{1}, Q_{2}>0$

Therefore, by differentiating $L$ we will get the following

$$
\begin{aligned}
& \dot{L}=-r_{1}^{T} P_{1} R r_{1}-r_{1}^{T} P_{1} K_{3} \int_{0}^{t} r_{1} d t+r_{1} P_{2} \int_{0}^{t} r_{1} d t-r_{2}^{T} Q_{1} S_{0} r_{2}- \\
& r_{2}^{T} Q_{1} C_{3} \int_{0}^{t} r_{2} d t+r_{2}^{T} Q_{2} \int_{0}^{t} r_{2} d t-r_{2}^{T} Q_{1} \beta \operatorname{sgn}\left(r_{2}\right)
\end{aligned}
$$

Define,

$$
\begin{gathered}
P_{2}=P_{1} K_{3} \\
Q_{2}=Q_{1} C_{3}
\end{gathered}
$$

then integration term vanishes.

$$
\dot{L}=-r_{1}^{T} P_{1} R r_{1}-r_{2}^{T} Q_{1} S_{0} r_{2}-r_{2}^{T} Q_{1} \beta \operatorname{sgn}\left(r_{2}\right)
$$

The Equation (55) can be written as

$$
\dot{L} \leq-\sigma_{\max }\left(P_{1} R\right) r_{1}^{2}-\sigma_{\max }\left(Q_{1} S_{0}\right) r_{2}^{2}-\sigma_{\max }\left(Q_{1} \beta\right)\left|r_{2}\right| \leq 0
$$


where, $\sigma_{\max }(\cdot)$ denotes the maximum singular value.

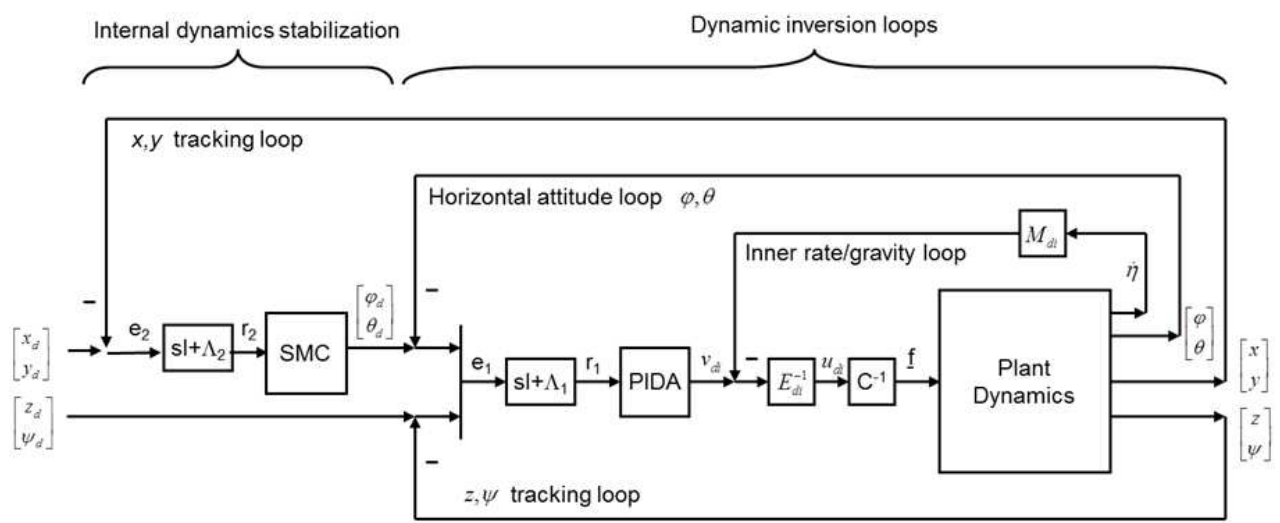

PIDA: Proportional Integral Derivative Control with Acceleration Feed forward SMC: Sliding Mode Control

Fig. 2. Control configuration

Comment 5.1: Equations (31)-(32) can also be rewritten as

$$
\begin{gathered}
\ddot{x}=-\frac{u}{m} \theta_{d}-\frac{u}{m}(-\tilde{\theta}) \\
\ddot{y}=\frac{u}{m} \varphi_{d}-\frac{u}{m} \tilde{\varphi}
\end{gathered}
$$

where $\tilde{\theta}=\theta_{d}-\sin \theta$ and $\tilde{\varphi}=\varphi_{d}-\cos \theta \sin \varphi$. According to (A. Das, K. Subbarao, and F. Lewis 2009) there exist a robustifying term $V_{r}$ which would modify the $v_{d i}$ as

$$
v_{d i}=\left[\begin{array}{c}
v_{z} \\
v_{\varphi} \\
v_{\theta} \\
v_{\psi}
\end{array}\right]=\left[\begin{array}{c}
\ddot{z}_{d}-K_{1_{z}}\left(\dot{z}-\dot{z}_{d}\right)-K_{2_{z}}\left(z-z_{d}\right) \\
\ddot{\varphi}_{d}-K_{1_{\varphi}}\left(\dot{\varphi}-\dot{\varphi}_{d}\right)-K_{2_{\varphi}}\left(\varphi-\varphi_{d}\right) \\
\ddot{\theta}_{d}-K_{1_{\theta}}\left(\dot{\theta}-\dot{\theta}_{d}\right)-K_{2_{\theta}}\left(\theta-\theta_{d}\right) \\
\ddot{\psi}_{d}-K_{1_{\psi}}\left(\dot{\psi}-\dot{\psi}_{d}\right)-K_{2_{\psi}}\left(\psi-\psi_{d}\right)
\end{array}\right]+V_{r}
$$

and thereby one can easily show that $\dot{L} \leq 0$ by suitbale choice of $V_{r}$. For this book chapter we considered the Eq. (36),(37), whcih in fact a simpler version of (31),(32). But we belive, for designing autopilot for quadrotor, the proposed mehtod discussed in this chapter can be used without loss of any genreality.

\section{Simulation results}

\subsection{Rotorcraft parameters}

Simulation for a typical quadrotor is performed using the following parameters (SI unit): 


$$
M_{1}=\left[\begin{array}{ccc}
1 & 0 & 0 \\
0 & 1 & 0 \\
0 & 0 & 1
\end{array}\right] ; \quad J=\left[\begin{array}{ccc}
5 & 0 & 0 \\
0 & 5 & 0 \\
0 & 0 & 15
\end{array}\right] ; \quad g=9.81
$$

\subsection{Reference trajectory generation}

As outlined in Refs (Hogan 1984; Flash and Hogan 1985), a reference trajectory is derived that minimizes the jerk (rate of change of acceleration) over the time horizon. The trajectory ensures that the velocities and accelerations at the end point are zero while meeting the position tracking objective. The following summarizes this approach:

$$
\dot{x}_{d}(t)=a_{1_{x}}+2 a_{2_{x}} t+3 a_{3_{x}} t^{2}+4 a_{4_{x}} t^{3}+5 a_{5_{x}} t^{4}
$$

Differentiating again,

$$
\ddot{x}_{d}(t)=2 a_{2_{x}}+6 a_{3_{x}} t+12 a_{4_{x}} t^{2}+20 a_{5_{x}} t^{3}
$$

As we indicated before that initial and final velocities and accelerations are zero; so from Eqs. (60) and (61) we can conclude the following:

$$
\left[\begin{array}{c}
d_{x} \\
0 \\
0
\end{array}\right]=\left[\begin{array}{ccc}
1 & t_{f} & t_{f}^{2} \\
3 & 4 t_{f} & 5 t_{f}^{2} \\
6 & 12 t_{f} & 20 t_{f}^{2}
\end{array}\right]\left[\begin{array}{c}
a_{3 x} \\
a_{4_{x}} \\
a_{5_{x}}
\end{array}\right]
$$

Where, $d_{x}=\left(x_{d_{f}}-x_{d_{0}}\right) / t_{f}^{3}$. Now, solving for coefficients

$$
\left[\begin{array}{l}
a_{3_{x}} \\
a_{4_{x}} \\
a_{5_{x}}
\end{array}\right]=\left[\begin{array}{ccc}
1 & t_{f} & t_{f}^{2} \\
3 & 4 t_{f} & 5 t_{f}^{2} \\
6 & 12 t_{f} & 20 t_{f}^{2}
\end{array}\right]^{-1}\left[\begin{array}{c}
d_{x} \\
0 \\
0
\end{array}\right]
$$

Thus the desired trajectory for the $x$ direction is given by

$$
x_{d}(t)=x_{d_{0}}+a_{3_{x}} t^{3}+a_{4_{x}} t^{4}+a_{5_{x}} t^{5}
$$

Similarly, the reference trajectories for the $y$ and $z$ directions are gives by Eq. (65) and Eq. (66) respectively.

$$
\begin{aligned}
& y_{d}(t)=y_{d_{0}}+a_{3_{y}} t^{3}+a_{4_{y}} t^{4}+a_{5_{y}} t^{5} \\
& z_{d}(t)=z_{d_{0}}+a_{3_{z}} t^{3}+a_{4_{z}} t^{4}+a_{5_{z}} t^{5}
\end{aligned}
$$

The beauty of this method lying in the fact that more demanding changes in position can be accommodated by varying the final time. That is acceleration/torque ratio can be controlled smoothly as per requirement. For example,

Let us assume at $t=0, x_{d_{0}}=0$ and at $t=10 \mathrm{sec}, x_{d_{f}}=10$. Therefore $d_{x}=0.01$ and the trajectory is given by Eq. (67) and shown in Fig. 3 for various desired final positions.

$$
x_{d}(t)=0.1 t^{3}-0.015 t^{4}+0.0006 t^{5}
$$




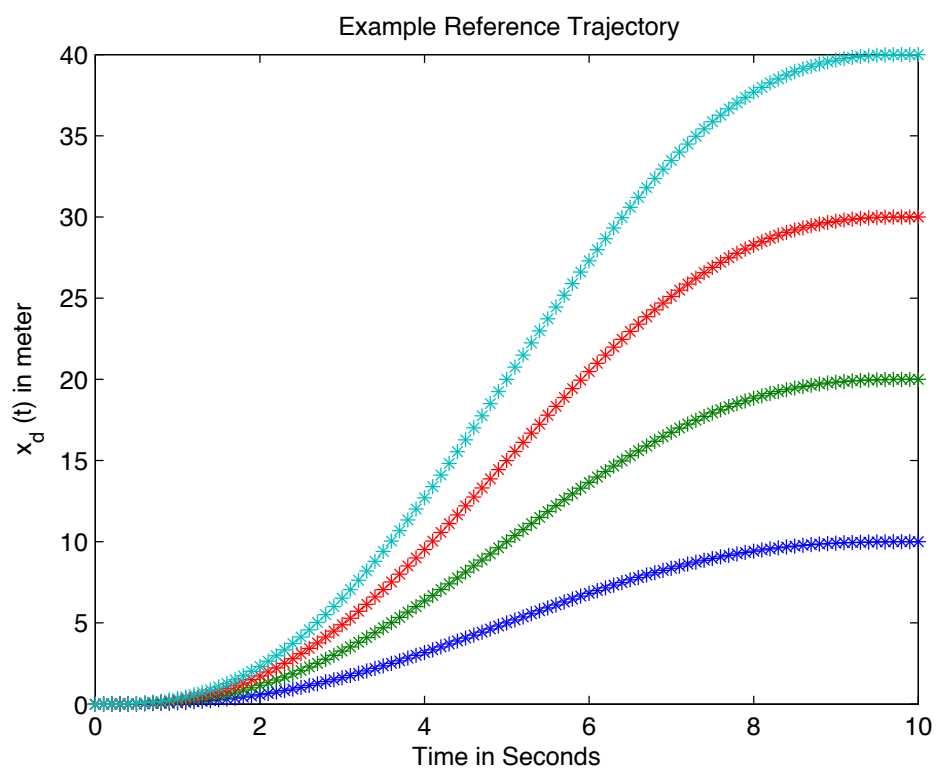

Fig. 3. Example trajectory simulation for different final positions

\subsection{Case 1: From initial position at $(0,5,10)$ to final position at $(20,-5,0)$}

Figure 4 describes the controlled motion of the quadrotor from its initial position $(0,5,10)$ to final position $(20,-5,0)$ for a given time (20 seconds). The actual trajectories $x(t), y(t), z(t)$ match exactly their desired values $x_{d}(t), y_{d}(t), z_{d}(t)$ respectively nearly exactly. The errors along the three axes are also shown in the same figure. It can be seen that the tracking is almost perfect as well as the tracking errors are significantly small. Figure 5 describes the attitude of the quadrotor $\varphi, \theta$ along with their demands $\varphi_{d}, \theta_{d}$ and attitude errors in radian. Again the angles match their command values nearly perfectly. Figure 6 describes the control input requirement which is very much realizable. Note that as described before the control requirement for yaw angle is $\tau_{\psi}=0$ and it is seen from Fig. 6.

\subsection{Case 2: From initial position at $(0,5,10)$ to final position at $(20,5,10)$}

Figures 7-8 illustrates the decoupling phenomenon of the control law. Fig. 7 shows that $x(t)$ follows the command $x_{d}(t)$ nearly perfectly unlike $y(t)$ and $z(t)$ are held their initial values. Fig. 8 shows that the change in $x$ does not make any influence on $\varphi$. The corresponding control inputs are also shown in Fig. 9 and due to the full decoupling effect it is seen that $\tau_{\varphi}$ is almost zero.

The similar type of simulations are performed for $y$ and $z$ directional motions separately and similar plots are obtained showing excellent tracking. 

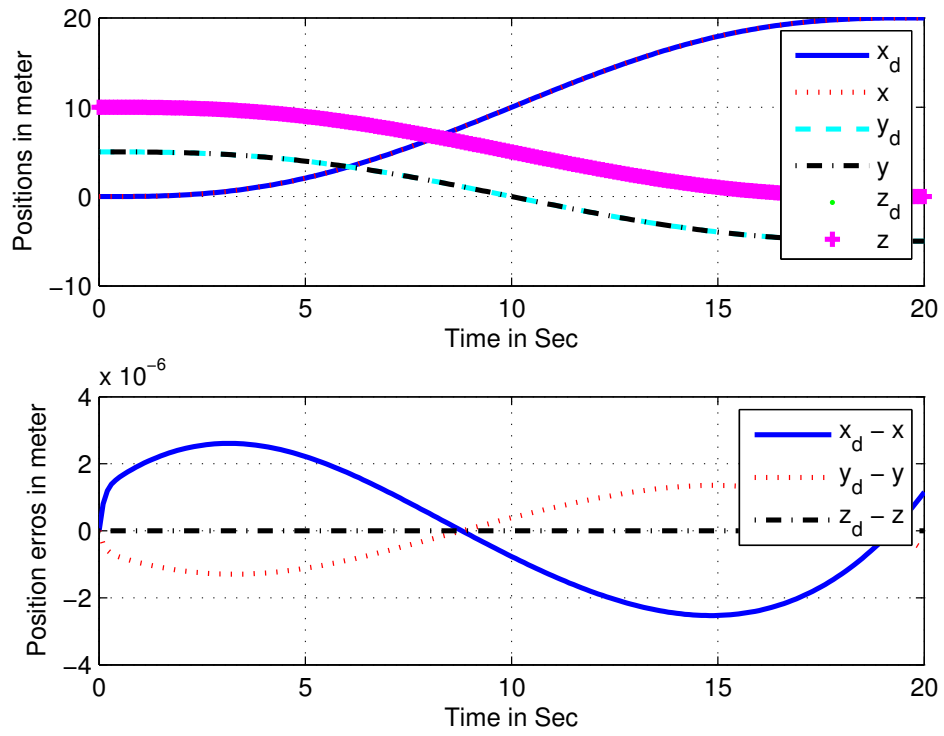

Fig. 4. Three position commands simultaneously
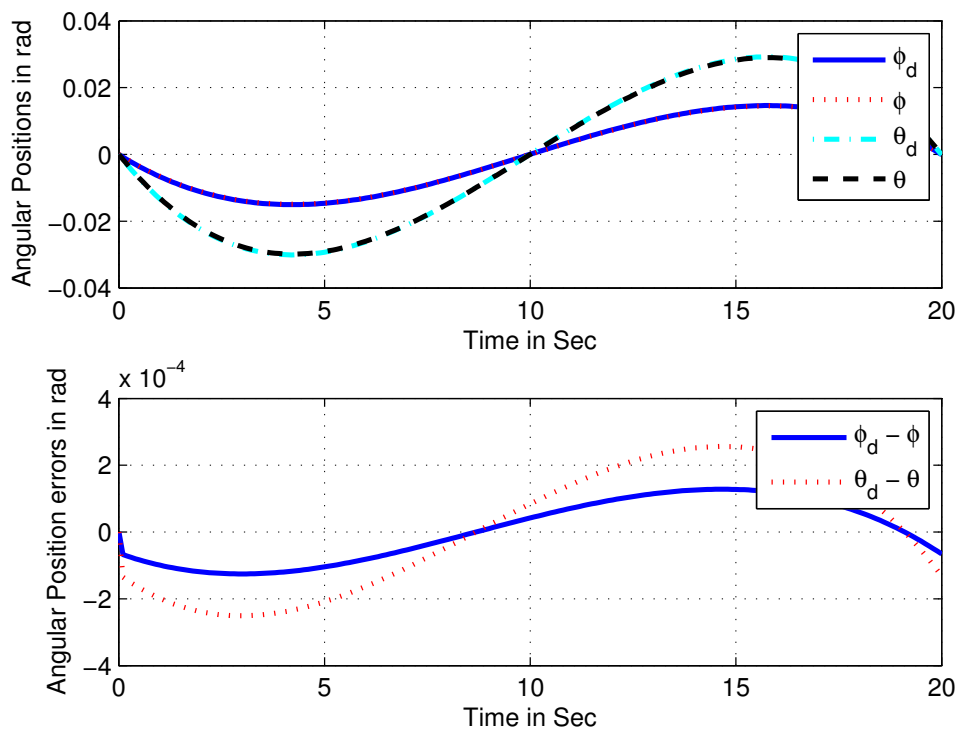

Fig. 5. Resultant angular positions and errors 

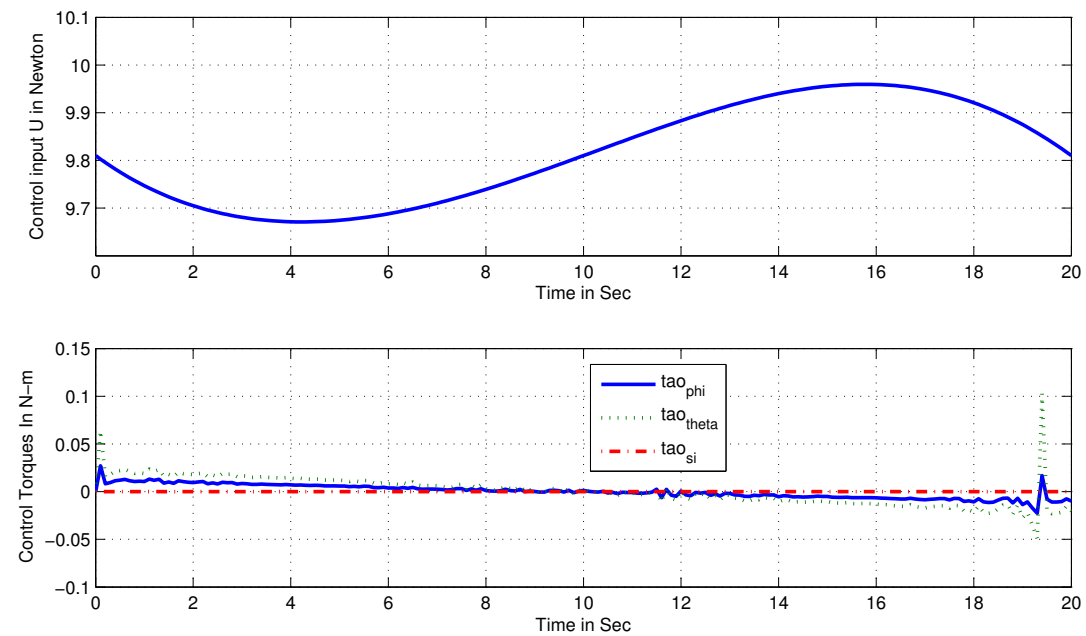

Fig. 6. Input commands for Case I
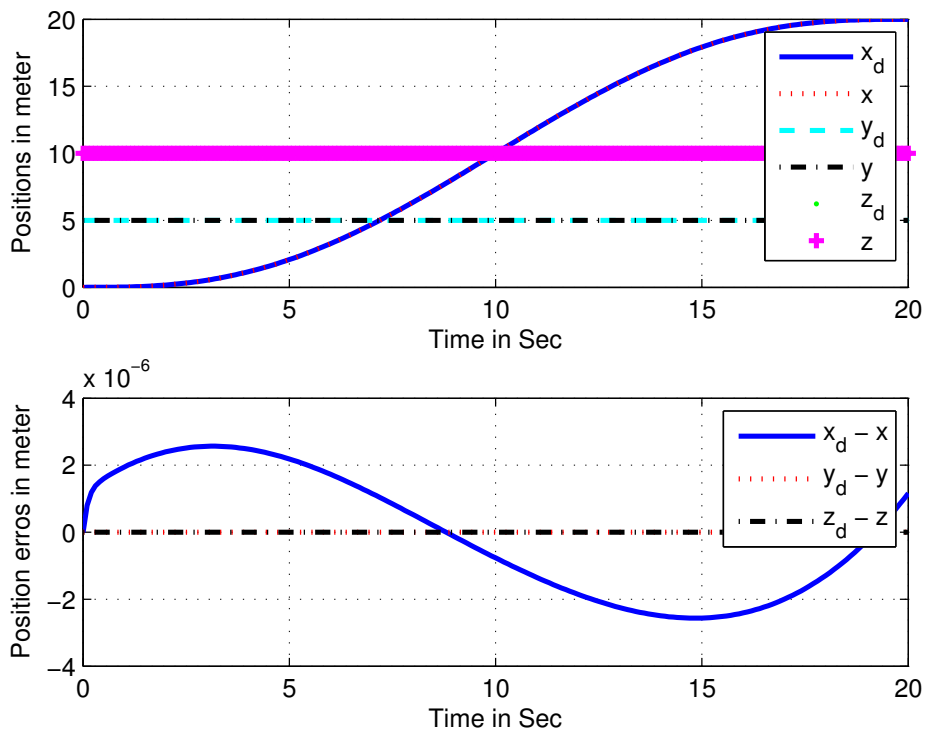

Fig. 7. Plots of position and position tracking errors for $x$ command only 

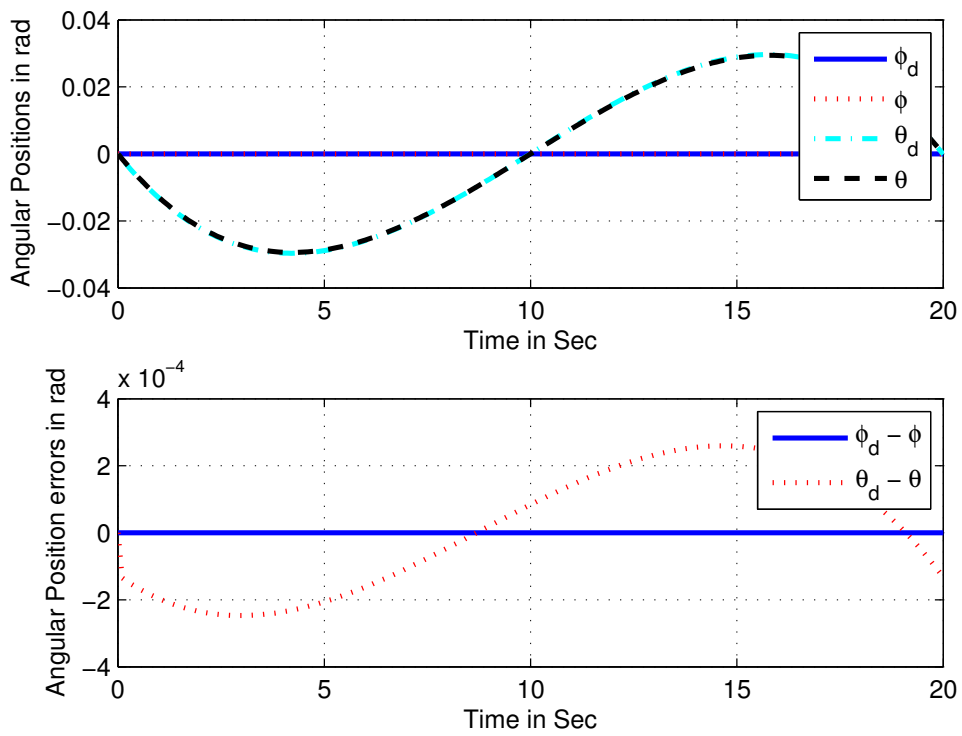

Fig. 8. Angular variations due to change in $x$
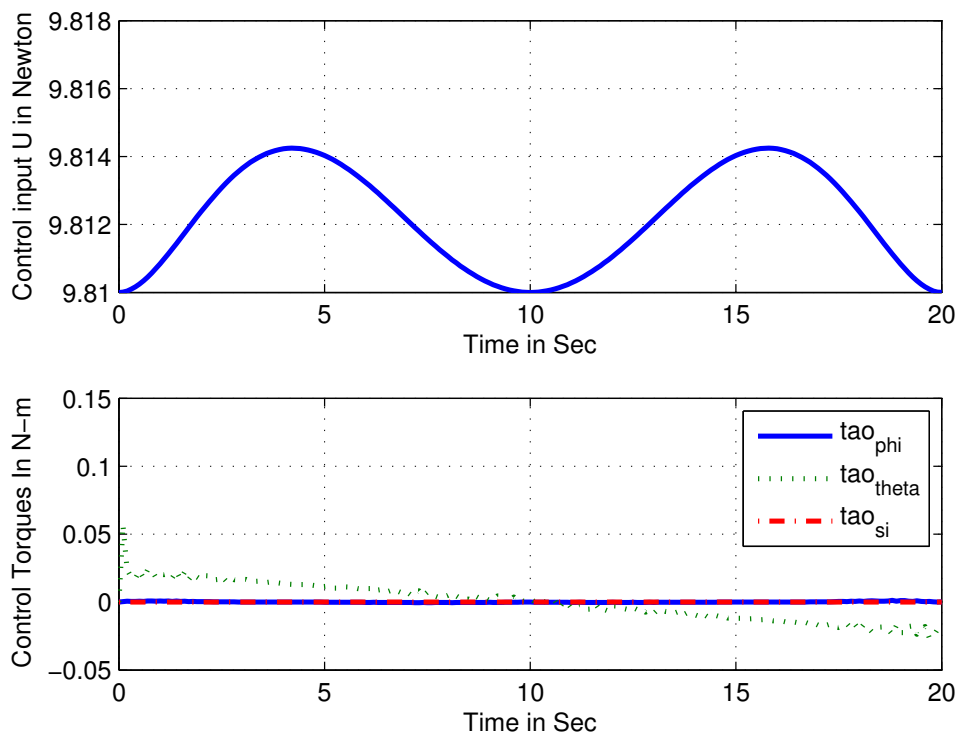

Fig. 9. Input commands for variation in $x$ (Case II) 


\subsection{Simulation with unmodeled input disturbances}

The simulation is performed to verify its robustness properties against unmodeled input disturbances. For this case we simulate the dynamics with high frequency disturbance $0.1^{*}$ $\sin (5 t)$ (1\% of maximum magnitude of force) for force channel and $0.01 \sin (5 t)(\sim 15 \%$ of maximum angular acceleration) for torque channel.

\subsection{Case-3: From initial position at $(0,5,10)$ to final position at $(20,-5,0)$ with disturbance}

Fig. 10-11 describes the motion of the quadrotor from its initial position $(0,5,10)$ to final position $(20,-5,0)$ for a given time (20 seconds) with input disturbances. It can be seen from Fig 10 that the quadrotor can track the desired position effectively without any effect of high input disturbances. From Fig 10 and Fig 11, it is also seen that the position errors are bounded and small. Fig. 12 shows the bounded variation of control inputs in presence of disturbance. Similar tracking performance is obtained for other commanded motion.
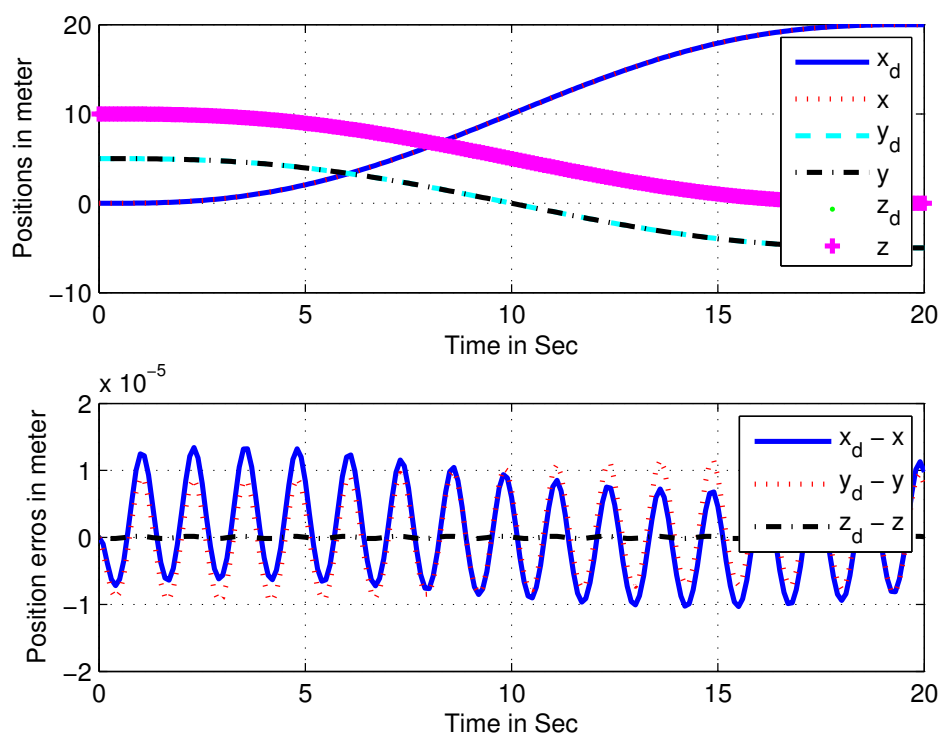

Fig. 10. Position tracking - Simultaneous command in $x, y$ and $z+$ Input disturbances 

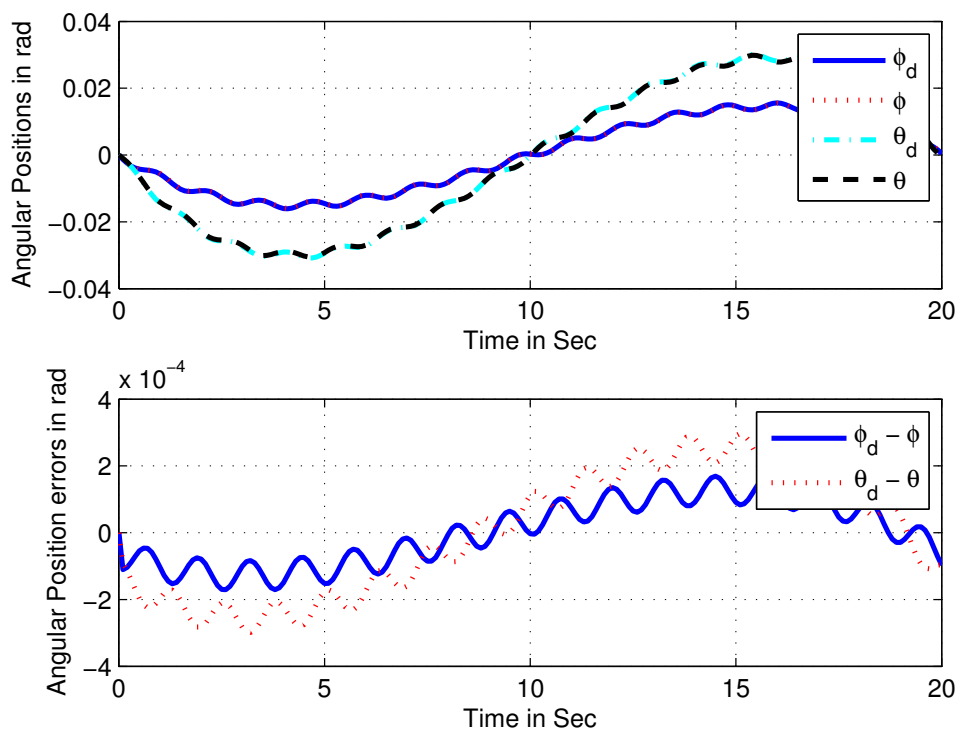

Fig. 11. Angular variations, errors and velocities (with input disturbances)
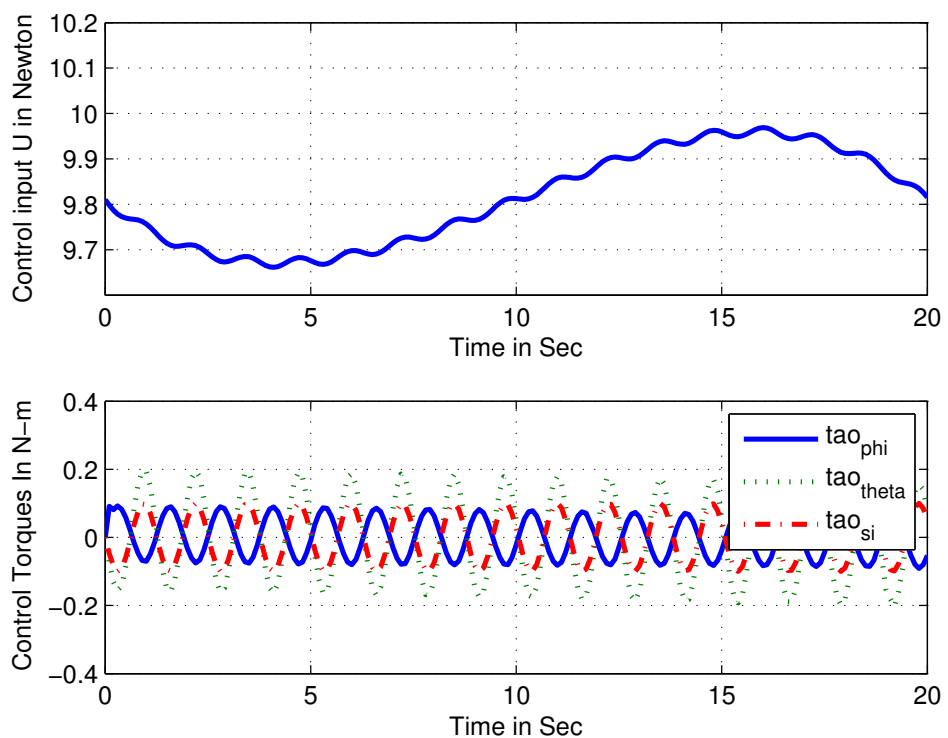

Fig. 12. Force and torque input variations (with input disturbances) 


\section{Conclusion}

Sliding mode approach using input-output linearization to design nonlinear controller for a quadrotor dynamics is discussed in this Chapter. Using this approach, an intuitively structured controller was derived that has an outer sliding mode control loop and an inner feedback linearizing control loop. The dynamics of a quadrotor are a simplified form of helicopter dynamics that exhibits the basic problems including under-actuation, strong coupling, multi-input/multi-output. The derived controller is capable of deal with such problems simultaneously and satisfactorily. As the quadrotor model discuss in this Chapter is similar to a full scale unmanned helicopter model, the same control configuration derived for quadrotor is also applicable for a helicopter model. The simulation results are presented to demonstrate the validity of the control law discussed in the Chapter.

\section{Acknowledgement}

This work was supported by the National Science Foundation ECS-0801330, the Army Research Office W91NF-05-1-0314 and the Air Force Office of Scientific Research FA9550-091-0278.

\section{References}

Altug, Erdinc, James P. Ostrowski, and Robert Mahony. 2002. Control of a Quadrotor Helicopter Using Visual Feedback ID - 376. In . Washington DC, Virginia, June.

B. Bijnens, Q. P. Chu, G. M. Voorsluijs, and J. A. Mulder. 2005. AIAA Guidance, Navigation, and Control Conference and Exhibit. In . San Francisco, California.

Bijnens, B., Q. P. Chu, G. M. Voorsluijs, and J. A. Mulder. 2005. Adaptive Feedback Linearization Flight Control for a Helicopter UAVID - 199.

Bouabdallah, Samir, AndrẤe Noth, and Roland Siegwart. 2004. International Conference on Intelligent Robots and Systems. In , 3:2451-2456. Sendal, Japan: IEEE.

Calise, A. J., B. S. Kim, J. Leitner, and J. V. R. Prasad. 1994. Helicopter adaptive flight control using neural networks. In . Lake Buena Vista, FL.

Campos, J., F. L. Lewis, and C. R. Selmic. 2000. Backlash Compensation in Discrete Time Nonlinear Systems Using Dynamic Inversion by Neural Networks. In . San Francisco, CA.

Castillo, P., A. Dzul, and R. Lozano. 2004. Real-time Stabilization and Tracking of a FourRotor Mini Rotorcraft. IEEE Transaction on Control System Technology 12: 510-516.

Castillo, P., R. Lozano, and A. Dzul. 2005. Modelling and Control of Mini Flying Machines. Springer-Verlag.

Das, A., T. Garai, S. Mukhopadhyay, and A. Patra. 2004. Feedback Linearization for a Nonlinear Skid-To-Turn Missile Model. First India annual conference, Proceedings of the IEEE INDICON 2004: 586-589.

Das, A., K. Subbarao, and F. Lewis. 2009. Dynamic inversion with zero-dynamics stabilisation for quadrotor control. Control Theory \& Applications, IET 3, no. 3 (March): 303 - 314. 
Das, Abhijit, Frank Lewis, and Kamesh Subbarao. 2009. Backstepping Approach for Controlling a Quadrotor Using Lagrange Form Dynamics. Journal of Intelligent and Robotic Systems 56, no. 1-2 (4): 127-151. doi:10.1007/s10846-009-9331-0.

Flash, T., and N. Hogan. 1985. The Coordination of Arm Movements: an Experimentally Confirmed Mathematical Model. Journal of Neuro Science 5: 1688-1703.

Gavrilets, V., B. Mettler, and E. Feron. 2003. Dynamic Model for a Miniature Aerobatic Helicopter. MIT-LIDS report LIDS-P-2580.

Hogan, N. 1984. Adaptive Control of Mechanical Impedance by Coactivation of Antagonist Muscles. IEEE Transaction of Automatic Control 29: 681-690.

Hovakimyan, N., F. Nardi, A. J. Calise, and H. Lee. 2001. Adaptive Output Feedback Control of a Class of Nonlinear Systems Using Neural Networks. International Journal of Control 74: 1161-1169.

Kanellakopoulos, I., P. V. Kokotovic, and A. S. Morse. 1991. Systematic Design of Adaptive Controllers for Feedback Linearizable Systems. IEEE Transaction of Automatic Control 36: 1241-1253.

Khalil, Hassan K. 2002. Nonlinear Systems. 3rd ed. Upper Saddle River, N.J: Prentice Hall.

Kim, B. S., and A. J. Calise. 1997. Nonlinear flight control using neural networks. Journal of Guidance Control Dynamics 20: 26-33.

Koo, T. J., and S. Sastry. 1998. Output tracking control design of a helicopter model based on approximate linearization. In Proceedings of the 37th Conference on Decision and Control. Tampa, Florida: IEEE.

Lewis, F., S. Jagannathan, and A. Yesildirek. 1999. Neural Network Control of Robot Manipulators and Nonlinear Systems. London: Taylor and Francis.

Mistler, V., A. Benallegue, and N. K. M'Sirdi. 2001. Exact linearization and non- interacting control of a 4 rotors helicopter via dynamic feedback. In 10th IEEE Int. Workshop on Robot-Human Interactive Communication. Paris.

Mokhtari, A., A. Benallegue, and Y. Orlov. 2006. Exact Linearization and Sliding Mode Observer for a Quadrotor Unmanned Aerial Vehicle. International Journal of Robotics and Automation 21: 39-49.

P. Castillo, R. Lozano, and A. Dzul. 2005. Stabilization of a Mini Rotorcraft Having Four Rotors. IEEE Control System Magazine 25: 45-55.

Prasad, J. V. R., and A. J. Calise. 1999. Adaptive nonlinear controller synthesis and flight evaluation on an unmanned helicopter. In . Kohala Coast-Island of Hawaii, USA.

Rysdyk, R., and A. J. Calise. 2005. Robust Nonlinear Adaptive Flight Control for Consistent Handling Qualities. IEEE Transaction of Control System Technology 13: 896-910.

Slotine, Jean-Jacques, and Weiping Li. 1991. Applied Nonlinear Control. Prentice Hall.

Stevens, B. L., and F. L. Lewis. 2003. Aircraft Simulation and Control. Wiley and Sons.

T. Madani, and A. Benallegue. 2006. Backstepping control for a quadrotor helicopter. In . Beijing, China. 
Wise, K. A., J. S. Brinker, A. J. Calise, D. F. Enns, M. R. Elgersma, and P. Voulgaris. 1999. Direct Adaptive Reconfigurable Flight Control for a Tailless Advanced Fighter Aircraft. International Journal of Robust and Nonlinear Control 9: 999-1012. 


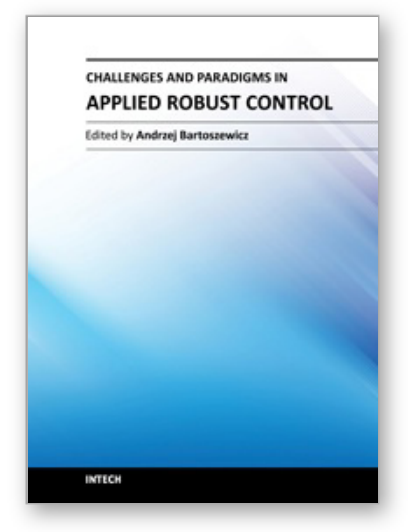

\author{
Challenges and Paradigms in Applied Robust Control \\ Edited by Prof. Andrzej Bartoszewicz
}

ISBN 978-953-307-338-5

Hard cover, 460 pages

Publisher InTech

Published online 16, November, 2011

Published in print edition November, 2011

The main objective of this book is to present important challenges and paradigms in the field of applied robust control design and implementation. Book contains a broad range of well worked out, recent application studies which include but are not limited to $\mathrm{H}$-infinity, sliding mode, robust PID and fault tolerant based control systems. The contributions enrich the current state of the art, and encourage new applications of robust control techniques in various engineering and non-engineering systems.

\title{
How to reference
}

In order to correctly reference this scholarly work, feel free to copy and paste the following:

Abhijit Das, Frank L. Lewis and Kamesh Subbarao (2011). Sliding Mode Approach to Control Quadrotor Using Dynamic Inversion, Challenges and Paradigms in Applied Robust Control, Prof. Andrzej Bartoszewicz (Ed.), ISBN: 978-953-307-338-5, InTech, Available from: http://www.intechopen.com/books/challenges-andparadigms-in-applied-robust-control/sliding-mode-approach-to-control-quadrotor-using-dynamic-inversion

\section{INTECH}

open science | open minds

\author{
InTech Europe \\ University Campus STeP Ri \\ Slavka Krautzeka 83/A \\ 51000 Rijeka, Croatia \\ Phone: +385 (51) 770447 \\ Fax: +385 (51) 686166 \\ www.intechopen.com
}

\author{
InTech China \\ Unit 405, Office Block, Hotel Equatorial Shanghai \\ No.65, Yan An Road (West), Shanghai, 200040, China \\ 中国上海市延安西路65号上海国际贵都大饭店办公楼 405 单元 \\ Phone: +86-21-62489820 \\ Fax: $+86-21-62489821$
}


(C) 2011 The Author(s). Licensee IntechOpen. This is an open access article distributed under the terms of the Creative Commons Attribution 3.0 License, which permits unrestricted use, distribution, and reproduction in any medium, provided the original work is properly cited. 